\section{Commentary: The best outcomes for pulmonary metastasectomy come from picking the right patients}

\author{
Alisa N. Blumenthaler, MD, ${ }^{\mathrm{a}}$ and \\ Mara B. Antonoff, MD, FACS ${ }^{b}$
}

The lungs are the most common site of metastasis in highgrade osteosarcoma, both at the time of initial diagnosis and on relapse after definitive treatment of the primary tumor. ${ }^{1,2}$ Aggressive surgical resection is believed to be the best chance for cure in patients with pulmonary metastases, and it is a standard component of the multimodality treatment approach. ${ }^{2,3}$ Long-term survival rates after pulmonary metastasectomy for osteosarcoma range from $30 \%$ to $40 \%$, although they can be higher in some subgroups of patients. ${ }^{2,4,5}$ As there have been no randomized trials to evaluate the benefit of pulmonary metastasectomy in patients with osteosarcoma, the evidential basis for this practice largely consists of retrospective studies aimed at identifying clinical and tumor-related prognostic variables. Unfortunately, due to the relatively rare incidence of this disease entity, many of the studies are limited by heterogeneous patient cohorts and small sample sizes.

The article by Liu and colleagues ${ }^{6}$ in this issue of the Journal aims to close this knowledge gap by using propensity-score based analyses to compare the outcomes of patients with pulmonary recurrence who were treated with metastasectomy and those who were not treated with metastasectomy. They also attempted to limit their analysis to a more homogenous patient group, including only patients with pulmonary relapse after surgical resection of a primary osteosarcoma who were deemed resectable and offered surgery. In both crude and propensity-score

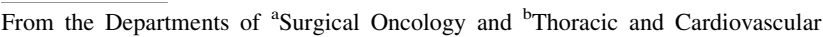
Surgery, University of Texas MD Anderson Cancer Center, Houston, Tex. Disclosures: The authors reported no conflicts of interest.

The Journal policy requires editors and reviewers to disclose conflicts of interest and to decline handling or reviewing manuscripts for which they may have a conflict of interest. The editors and reviewers of this article have no conflicts of interest.

Received for publication Nov 2, 2020; revisions received Nov 2, 2020; accepted for publication Nov 3, 2020; available ahead of print Nov 6, 2020.

Address for reprints: Mara B. Antonoff, MD, FACS, Department of Thoracic and Cardiovascular Surgery, University of Texas MD Anderson Cancer Center, 1515 Holcombe Blvd, Houston, TX 77030 (E-mail: MBAntonoff@mdanderson.org).

J Thorac Cardiovasc Surg 2022;163:483-4

$0022-5223 / \$ 36.00$

Copyright (c) 2020 by The American Association for Thoracic Surgery

https://doi.org/10.1016/j.jtcvs.2020.11.014
}

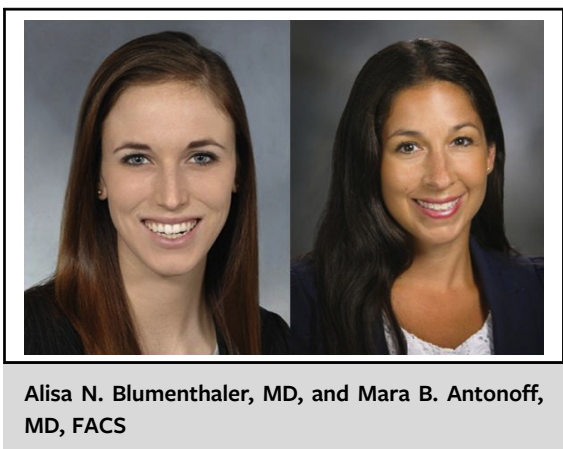

CENTRAL MESSAGE
Wide-ranging results for pulmo-
nary metastasectomy are seen in
heterogenous cohorts lacking
uniform application of appro-
priate selection criteria, whereas
optimal patient selection clearly
improves survival.

analyses, Liu and colleagues identify a significant postrelapse survival (PRS) benefit in the metastasectomy group. PRS rates at 2 and 5 years were $68.4 \%$ and $41.0 \%$, respectively, in the surgical group, compared with $25.0 \%$ and $0 \%$ in the nonsurgery group. Further analysis with 11 different propensity score models provide additional support for their findings.

Previous studies have identified numerous important prognostic factors in patients with osteosarcoma and pulmonary metastases, namely number of metastases, disease-free interval, primary tumor response to chemotherapy, presence of extrapulmonary disease, and features specific to the lung lesions, including size, bilateral spread, and pleural disruption. ${ }^{1,3-5,7}$ Notably, the completion of resection has also been identified as an important prognostic indicator, with patients who achieved a second complete remission after metastasectomy faring significantly better than those with incomplete remission. ${ }^{1,3,5}$ A secondary finding of Liu and colleagues corroborates these previous studies, identifying a survival benefit in the 55 patients with a complete surgical resection (32.3 months) compared with the 4 patients with an incomplete resection (14.4 months) and those not treated with surgery (13.8 months).

This prognostic factor underscores the importance of the resectability assessment that Liu and colleagues emphasize in their article. Patients for whom a complete remission is 
not achieved appear to be unlikely to derive a survival benefit from aggressive surgical resection. It is a testament to the appropriateness of their assessment algorithm that only 4 of 59 patients who underwent metastasectomy had an incomplete resection.

Liu and colleagues also evaluated subgroups based on clinical and tumor-related factors. They found that patients with pulmonary lesions in 3 or more lung lobes and those with very high (more than twice the upper limit of normal) serum alkaline phosphatase (ALP) levels at the time of primary diagnosis did not benefit from pulmonary metastasectomy. The assessment for surgical candidacy may be further improved by incorporating factors such as these to avoid operations in patients that may be better served by nonoperative treatments.

A notable strength of this article is that the authors made an effort to include a homogenous group of patients, including only those who had recurrence after primary tumor resection, and only patients who were deemed resectable by their multidisciplinary team. The selection bias inherent in retrospective analyses may be minimized, as all patients included in the study were prospectively determined to be operable candidates, based on a predetermined resectability assessment algorithm used by their multidisciplinary team. The authors should be commended for their extensive efforts to control for confounding biases in this retrospective study by creation and utilization of multiple statistical models. However, it is important to note limitations remain despite rigorous statistical confounding bias control, namely that due to the nature of a retrospective study and unavailable data, the authors were not able to control for the extent of the resection required, comorbidities, or pulmonary function tests.
Furthermore, in the unmatched group, the disease-free interval, size of pulmonary metastases, and rates of relapse during adjuvant chemotherapy are significantly different between the 2 groups, which, despite all patients being metastasectomy candidates, may represent a difference in tumor biology that cannot be fully captured in the propensity score analyses. Nevertheless, this article contributes further evidence in support of metastasectomy for pulmonary recurrence in osteosarcoma, while identifying subgroups of patients who may benefit more from treatment with novel agents rather than aggressive surgical resection. Ultimately, it is clear that when appropriate selection criteria are applied, pulmonary metastasectomy can improve survival outcomes, and in a world where biology determines all, picking the right patients for surgery will always be key.

\section{References}

1. Kempf-Bielack B, Bielack SS, Jürgens H, Branscheid D, Berdel WE, Exner GU, et al. Osteosarcoma relapse after combined modality therapy: an analysis of unselected patients in the Cooperative Osteosarcoma Study Group (COSS). J Clin Oncol. 2005;23:559-68.

2. Meazza C, Scanagatta P. Metastatic osteosarcoma: a challenging multidisciplinary treatment. Expert Rev Anticancer Ther. 2016;16:543-56.

3. Harting MT, Blakely ML. Management of osteosarcoma pulmonary metastases. Semin Pediatr Surg. 2006;15:25-9.

4. Harting MT, Blakely ML, Jaffe N, Cox CS Jr, Hayes-Jordan A, Benjamin RS, et al. Long-term survival after aggressive resection of pulmonary metastases among children and adolescents with osteosarcoma. J Pediatr Surg. 2006;41:194-9.

5. Iwata S, Yonemoto T, Iizasa T, Niibe Y, Kamoda H, Ishii T. Oligo-recurrence of osteosarcoma patients: treatment strategies for pulmonary metastases. Ann Surg Oncol. 2015;22(Suppl 3):S1332-8.

6. Liu Z, Yin J, Zhou Q, Yang J, Zeng B, Yeung S-CJ, et al. Survival after pulmonary metastasectomy for relapsed osteosarcoma. J Thorac Cardiovasc Surg. 2022;163: 469-79.e8.

7. Ahmed G, Zamzam M, Kamel A, Ahmed S, Salama A, Zaki I, et al. Effect of timing of pulmonary metastasis occurrence on the outcome of metastasectomy in osteosarcoma patients. J Pediatr Surg. 2019;54:775-9. 